\title{
The Semicolon Sign: Dopamine Transporter Imaging Artifact from Head Tilt
}

\author{
Matthew F. Covington, Natalie A. McMillan, Ryan J. Avery, and Phillip H. Kuo
}

Department of Medical Imaging, University of Arizona, Tucson, Arizona

\begin{abstract}
Dopamine transporter (DAT) imaging is a valuable tool to aid in the diagnosis of Parkinson disease and other Parkinsonian syndromes. DAT imaging is special among clinical nuclear medicine scans in that the already small caudate and putamen are presented in multiple thin axial cuts. Because the imaged basal ganglia are small, slight differences in head tilt may result in a significant artifact that we have termed the semicolon sign. The semicolon sign occurs when forward head tilt creates select images that show the caudate nuclei separate from the putamen. This gives the false impression that DAT activity in the putamen is decreased or absent. To avoid falsely attributing this artifact to loss of putaminal activity, it is imperative that the interpreting physician first recognize the artifact and then mentally integrate all provided images to identify normal activity in the putamen on subsequent levels. Furthermore, quantitative software packages for automated DAT scan interpretation are now available. If images demonstrating the semicolon sign are used for automated interpretation, loss of activity in the putamen may be falsely calculated, thereby contributing to erroneous results. Quality control measures are essential to ensure that technologists correctly position each patient's head to minimize head tilt artifact on DAT scan images. A protocol to obtain optimal head positioning is presented.
\end{abstract}

Key Words: dopamine transporter (DAT) imaging; imaging artifact; basal ganglia; Parkinson disease

J Nucl Med Technol 2013; 41:105-107

DOI: 10.2967/jnmt.112.117184

D opamine transporter (DAT) imaging is a valuable tool to aid in the diagnosis of Parkinson disease and other Parkinsonian syndromes such as Lewy body dementia and progressive supranuclear palsy $(1,2)$. DAT imaging is special among clinical nuclear medicine scans in that the already small caudate and putamen, with average volumes of 3.4 and $4.3 \mathrm{~cm}^{3}$, respectively (3), are presented in multiple thin axial cuts. Because the imaged basal ganglia are small, slight differences in head tilt may result in a significant artifact (Fig. 1).

For correspondence or reprints contact: Phillip H. Kuo, 1501 N. Campbell Ave., P.O. Box 245067, Tucson, AZ 85724-5067.

E-mail: philliphkuo@gmail.com

Published online Mar. 21, 2013.

COPYRIGHT (C) 2013 by the Society of Nuclear Medicine and Molecular Imaging, Inc.
We have coined the term semicolon sign for the characteristic artifact from frontal head tilt. This nomenclature is in keeping with the "commas" and "periods" commonly used to describe the normal and abnormal appearance of the basal ganglia in Parkinsonian syndromes, respectively. The semicolon sign occurs when forward head tilt creates select images that show the caudate nuclei separate from the putamen (Fig. 2). This may give the false impression that DAT activity in the putamen is decreased or absent. Therefore, the physician may provide an incorrect diagnosis of dopaminergic deficiency, with potential negative clinical consequences.

To avoid falsely attributing this artifact to loss of putaminal activity, the interpreting physician must first recognize the artifact and then mentally integrate all provided images to identify normal activity in the putamen on subsequent levels. Quality control measures are essential to ensure that technologists correctly position each patient's head to minimize head tilt artifact on DAT scan images. If technologists readily recognize this artifact, they can potentially rescan the patient in a more optimal position. According to consensus practice guidelines (4), the technologist may alternatively use widely available software packages to correct the head tilt artifact by manipulating the orientation of acquired axial images. Such head tilt correction may be particularly useful for patients who are unable to maintain proper head positioning because of discomfort or illness. Software packages are also available for quantitative automated DAT scan interpretation $(5,6)$. These rely on axial images for automated analysis. If images demonstrating the semicolon sign are used for automated interpretation, loss of activity in the putamen may be falsely calculated, thereby contributing to erroneous results.

Proper head positioning may be obtained by the following protocol. A head and neck holder should be used with the head resting entirely within the corresponding concavity of the holder and the vertex of the head reaching the head holder's superior edge (Fig. $3 \mathrm{~A})$. Failure to position the head properly within the head holder may cause forward head tilt (Fig. 3B). Next, the canthomeatal line (imaginary line from the lateral corner of the eye to the ear canal) should be identified and oriented as vertically as possible. Even small deflections of the canthomeatal line from the 
FIGURE 1. Effect of head positioning on axial imaging of caudate (gold) and putamen (blue). These images represent rough approximation of basal ganglia anatomy. (A) Abnormal forward head tilt results in slices (red lines) through caudate head that do not include putamen. Subsequent slices (white lines) demonstrate both caudate and putamen. (B) With correct head positioning, all slices through caudate head also include putamen (white lines).

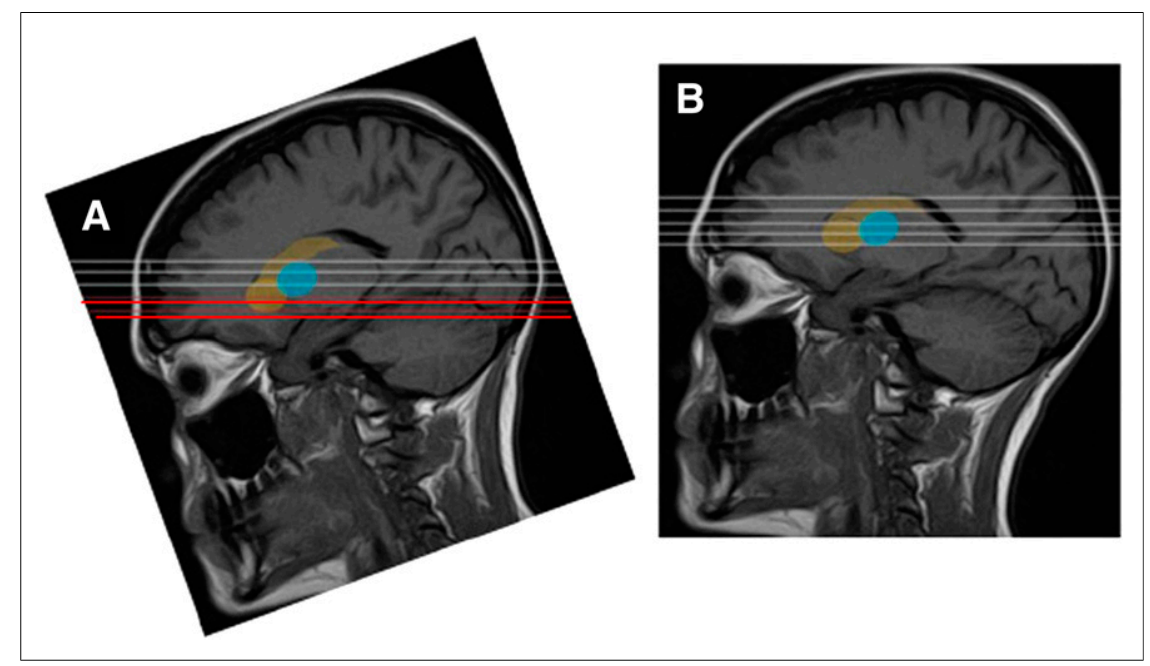

vertical position may signify abnormal head tilt. Chin position is also a useful measure of head tilt. The chin should lie in a neutral position when viewed from the side. Deflection of the chin toward the neck indicates abnormal forward head tilt. Finally, the technologist should stand at the head of the patient's bed and examine the vertex, ears, and nose to correct any signs of lateral head rotation.
FIGURE 2. Semicolon sign. Abnormal forward head tilt creates images in which caudate head is imaged in separate axial slices from putamen. (Images are obtained with filtered backprojection using Butterworth filter with cutoff of 0.6 and order of 8 . No attenuation correction was applied. Matrix size of $128 \times 128$ was acquired at 1.23 zoom and was manually adjusted to zoom of 1.6. Axial images are presented at slice thickness of $2.5 \mathrm{~mm}$. Images were acquired at $30 \mathrm{~s} /$ frame over 30-min acquisition time using dual-head camera. Circular orbit was used with radius of $13 \mathrm{~cm}$. Stop-and-shoot setting was $3^{\circ}$. Bottom edge of field of view is positioned at level of patient's nose). (A) Top 2 rows demonstrate normal appearance of properly aligned caudate heads and putamen with comma appearance. Bottom 2 rows demonstrate semicolon artifact in which caudate heads appear separate from putamen on isolated slices. (B) This graphic depicts separate axial images of caudate heads and putamen that have been superimposed to illustrate semicolon appearance.
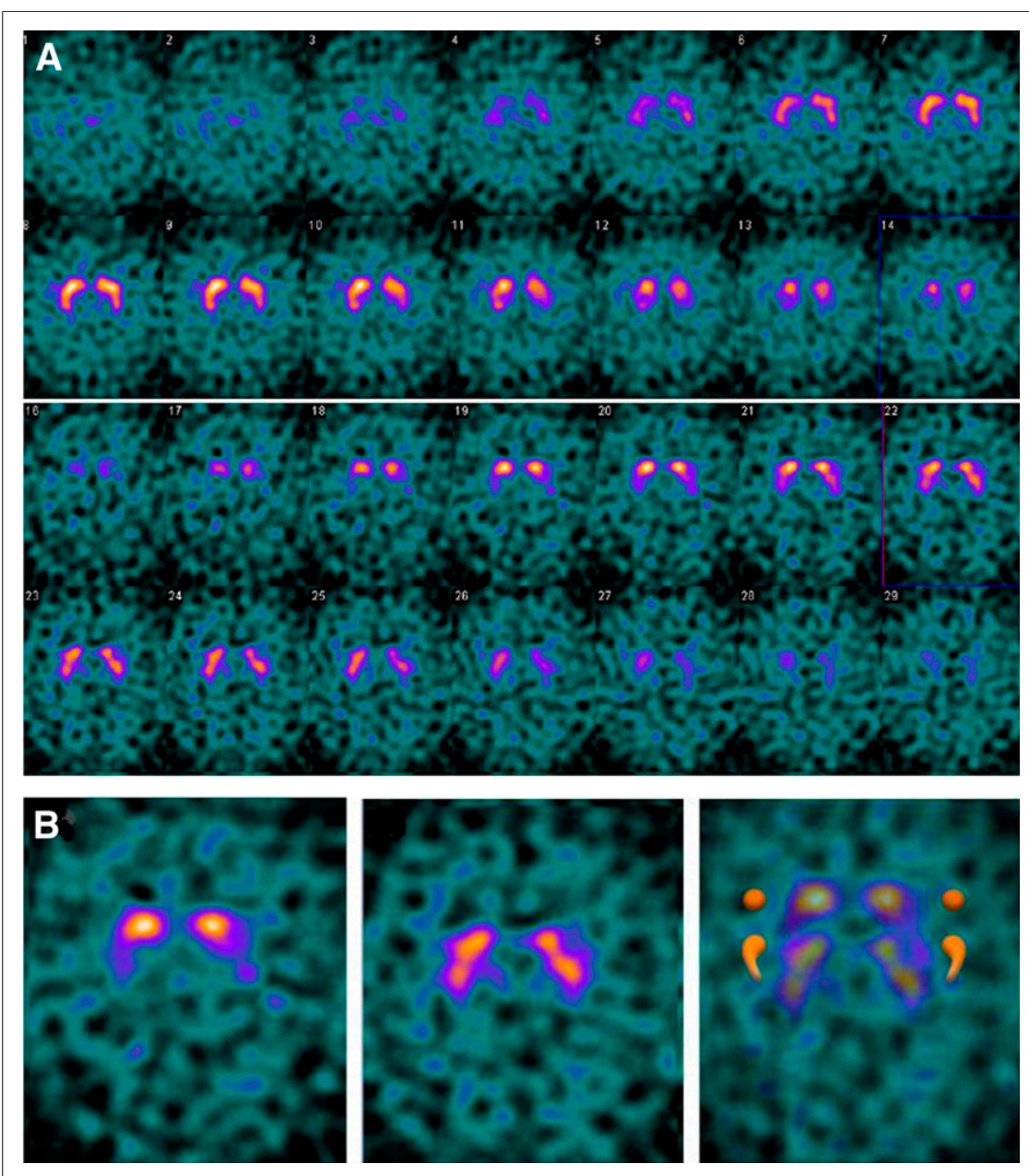


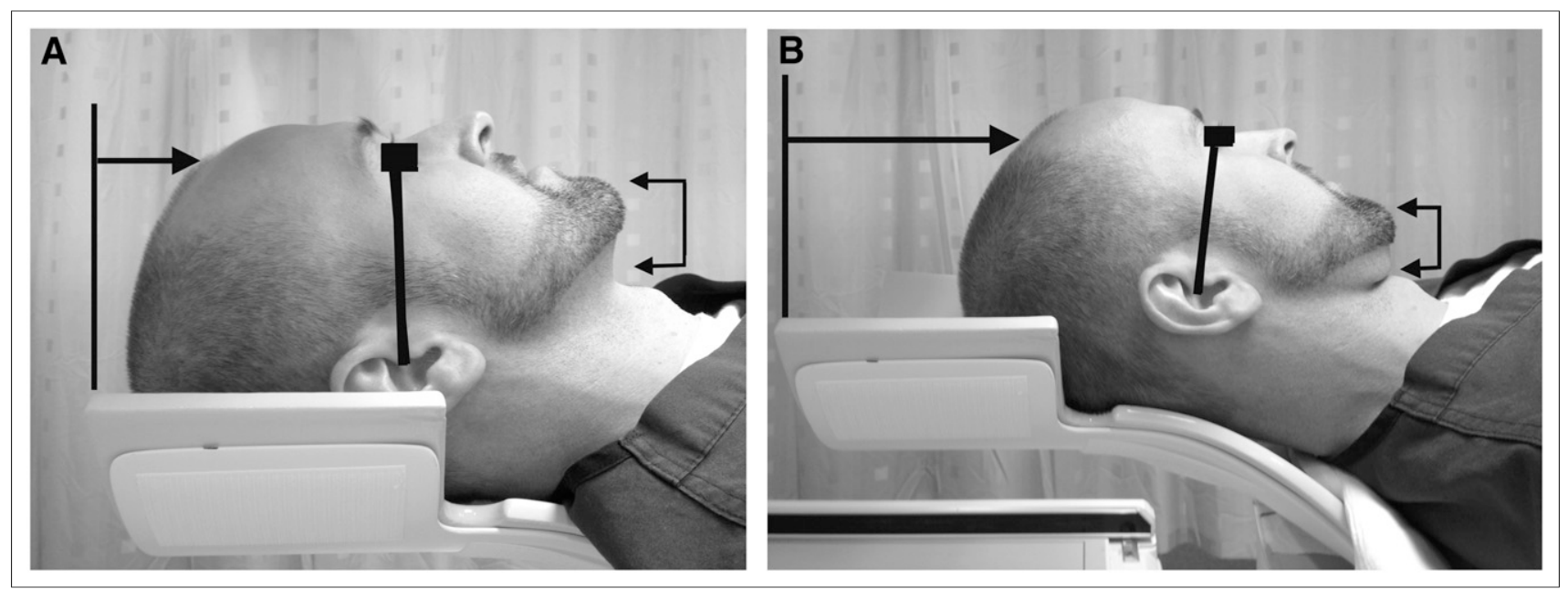

FIGURE 3. Correct head positioning using head holder (Composites Horizons, Inc.). (A) Vertex of head should reach head holder's superior edge (black line with arrow). Canthomeatal line should be oriented as vertically as possible (black line). Chin should rest in neutral position (connected arrowheads). (B) Incorrect positioning of head within head holder may result in head tilt artifact. Vertex of head does not reach head holder's superior edge (black line with arrow). Canthomeatal line (black line) is slightly deflected forward, indicating abnormal forward head tilt. Chin is also deflected toward neck (connected arrowheads).

\section{REFERENCES}

1. Ravina B, Marek K, Eberly S, et al. Dopamine transporter imaging is associated with long-term outcomes in Parkinson's disease. Mov Disord. 2012;27: 1392-1397.

2. Varrone A, Marek KL, Jennings D, Innis RB, Seibyl JP. $\left[{ }^{123}\right.$ I]beta-CIT SPECT imaging demonstrates reduced density of striatal dopamine transporters in Parkinson's disease and multiple system atrophy. Mov Disord. 2001;16: $1023-1032$.
3. Gunning-Dixon FM, Head D, McQuain J, Acker JD, Raz N. Differential aging of the human striatum: a prospective MR imaging study. AJNR. 1998;19:1501-1507. 4. Djang DSW, Janssen MJR, Bohnen N, et al. SNM practice guideline for dopamine transporter imaging with 123 I-ioflupane SPECT 1.0. J Nucl Med. 2012;53:154-163.

5. Tatsch K, Poepperl G. Quantitative approaches to dopaminergic brain imaging. Q J Nucl Med Mol Imaging. 2012;56:27-38.

6. Zubal IG, Early M, Yuan O, et al. Optimized, automated striatal uptake analysis applied to SPECT brain scans of Parkinson's disease patients. J Nucl Med. 2007;48:857-864. 